\title{
Cryo-EM structures of inhibitory antibodies complexed with Arginase1 provide insight into mechanism of action.
}

\author{
Rachel Palte ${ }^{1}$, Veronica Juan ${ }^{2}$, Yacob Gomez-Llorente ${ }^{3}$, Giovanna Scapin ${ }^{4}$, Marc Bailly ${ }^{5}$, Laurence \\ Fayadat-Dilman $^{6}$, Symon Gathiaka ${ }^{7}$, Brian Hall, ${ }^{8}$ Mas Handa ${ }^{9}$, Esther Kofman ${ }^{10}$, Mark Hsieh ${ }^{11}$, J. \\ Richard Miller ${ }^{12}$, Shane Taremi ${ }^{13}$ \\ ${ }^{1}$ Merck \& Co. ${ }^{2}$ Merck Research Labs, ${ }^{3}$ Merck Research Labs, ${ }^{4}$ Nanolmaging Services, ${ }^{5}$ Merck \\ Research Labs, ${ }^{6}$ Merck Research Labs, ${ }^{7}$ Merck Research Labs, ${ }^{8}$ Merck Research Labs, ${ }^{9}$ Merck \\ Research Labs, ${ }^{10}$ Merck Research Labs, ${ }^{11}$ Merck Research Labs, ${ }^{12}$ Merck Research Labs \\ ${ }^{13}$ Merck Research Labs \\ rachel.kubiak@merck.com
}

Human Arginase 1 (hArg1) is a metalloenzyme that catalyzes the hydrolysis of L-arginine to L-ornithine and urea and modulates T-cell-mediated immune response. Arginase-targeted therapies have been pursued across several disease areas including immunology, oncology, nervous system dysfunction, and cardiovascular dysfunction and diseases. Currently, all published hArg1 inhibitors are small molecules usually less than $350 \mathrm{Da}$ in size. Utilizing cryo-electron microscopy we have obtained structures of potent and inhibitory anti-hArg antibodies bound to hArg1. These distinct macromolecular complexes are made up of multiple hArg and $\mathrm{mAb}$ molecules and are greater than $650 \mathrm{kDa}$ in size. With local resolutions of $3.5 \AA$ or better we unambiguously mapped epitopes and paratopes for all five antibodies and determined that the antibodies act through both orthosteric and allosteric mechanisms. These hArg1:antibody complexes for the first time present an alternative mechanism to inhibit hArg1 activity and highlight the ability to utilize antibodies as probes in the discovery and development of peptide and small molecule inhibitors for enzymes in general.

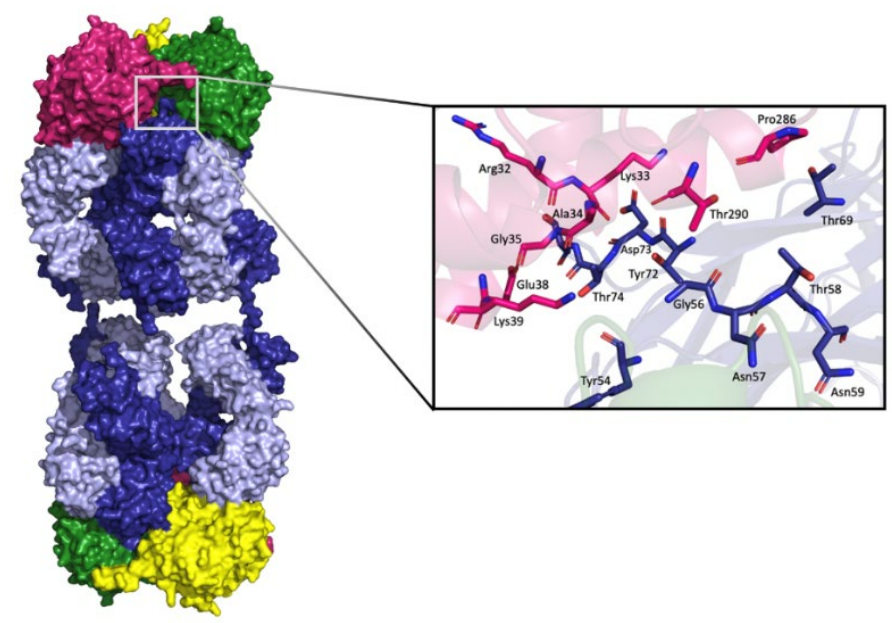

Figure 1 